\title{
Transit \& RV Observations of Exoplanets by the 1-m Telescope at Weihai
}

\author{
Chen Cao ${ }^{1}$, Dayong Ren ${ }^{1}$, Dongyang Gao ${ }^{1}$, Jicheng Zhang ${ }^{1,2}$, \\ Nan Song ${ }^{2}$ and Feige Wang ${ }^{3}$ \\ ${ }^{1}$ College of Space Science and Physics, Shandong University at Weihai, \\ Cultural West road No.180, Weihai, Shandong, China \\ email: caochen@sdu.edu.cn \\ ${ }^{2}$ National Astronomical Observatories, Chinese Academy of Sciences, Beijing, China \\ ${ }^{3}$ Dept. of Astronomy, Peking University, Beijing, China
}

\begin{abstract}
By using the 1-m reflecting telescope at Weihai Observatory of Shandong University, the transit observations of seven stars are carried out to accurately estimate the physical parameters of extrasolar planets. Besides, a new high-resolution spectrograph (WES) was installed on this telescope for radial velocity measurements on exoplanets, we will show some preliminary results.
\end{abstract}

Keywords. planetary systems, techniques: photometric, techniques: radial velocities

\section{Introduction}

In recent years a lot of new exoplanets were discovered by using accurate photometric transit method and radial velocity measurements with very high precision. Especially for detecting habitable (super)earth, the Kepler mission made a great progress (see, e.g., Borucki et al. 2010 and other Kepler papers). Although many of the great discoveries were made by world's largest telescopes and space-based observatories, using groundbased and small-size telescopes we can still make significant contribution to this study, and also very useful for undergraduates' education and public outreach on exoplanets.

Weihai observatory of Shandong University (WHO) was founded in 2007, it is located at the beautiful seaside city Weihai of Shandong province in east China, and managed by College of Space Science and Physics, Shandong University at Weihai. WHO has a one meter diameter reflecting telescope made by APM company, Germany, it can be used for photometry and imaging with a scientific 2k*2k CCD (PI PIXIS-2048B) and broadband filters (UBVRI and SDSS ugriz), and high-resolution spectroscopic observations with a fiber-fed echelle spectrograph (WES).

\section{Transit Observations}

Using 1-m telescope at WHO, we made several transit observations on seven stars which are known to have planetary systems: TrES-1, TrES-3, XO-2, WASP-1, WASP2, WASP-3 and HAT-P-7. The data were then reduced using basic aperture photometry procedures by IRAF and MaxIM DL, and submitted to the Exoplanet Transit Database (ETD,Poddaný, Brát \& Pejcha 2010). Most of the observations have reference photometric accuracies better than 3mmag. A simple analytical model was used to calculate the central time, duration and depth of the transit according to ETD. Then the physical parameters of exoplanets (e.g., planet radius) were estimated and compared with previous results. Fig. 1 gives an example of the transit light curves we 
observed (for TrES-3b). For more details please see: Zhang et al. 2011 and the ETD (http://var2.astro.cz/EN/tresca/transits.php, search for Chen CAO's observations). Our transit observations can be used for better estimates of planetary parameters, and also for searching additional third bodies using transit timing analysis (e.g., Rabus et al. 2009).

In addition, we also try to use another $30-\mathrm{cm}$ refracting telescope and an educationalclass CCD (SBIG ST-8) to do such transit observations. Although the photometric accuracies are worse than our 1-m results, we still successfully observed the transit of HD189733b (for details please see ETD and search for Nan SONG's observations).

\section{Radial Velocity Observing System}

In 2010, a new high-resolution echelle spectrograph (WES) was installed on our 1$\mathrm{m}$ telescope, it is the first fiber-fed high-resolution spectrograph in China. The spectral resolution is designed to be $\mathrm{R} \sim 40000-60000$, and it can gain $\mathrm{S} / \mathrm{N} \sim 100$ for $\operatorname{mag}(\mathrm{V})=8$ stars for one hour exposure. The spectrograph is also equipped with an iodine cell for high precision radial velocity (RV) measurements. Using a RV standard star HD113226, we estimated that the short-period RV precision is better than $\sim 10 \mathrm{~m} / \mathrm{s}$, shown that our RV observing system can be used to detect giant exoplanets. Fig. 2 shows part of the observed spectra of a bright star Alkaid (HIP67301), overlapped with I2 absorption features. For more details about WES, please see Dongyang Gao's paper in this symposium. The WES with RV observing system will join the East Asia exoplanet searching network (EAPSNET), collaborate with exoplanet hunters from China, Japan and Korea (e.g., Wang et al. 2012, Liu et al. 2009, Sato et al. 2008), to search for the new worlds around giant and dwarf stars.

Besides, we also try to use WES to make transmission spectra test observations on transiting exoplanets, such as HD189733b. Previous results made by HET (Redfield et al.

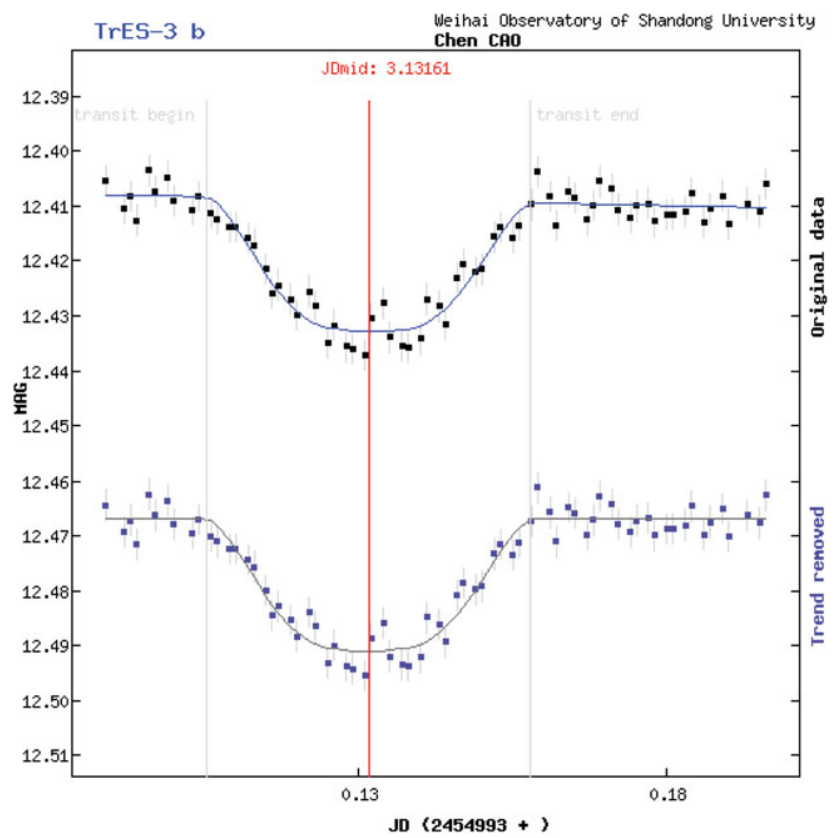

Figure 1. An example (TrES-3b) of our transit observations made by 1-m telescope. For more details please see: Zhang et al. 2011 and the ETD (http://var2.astro.cz/EN/tresca/transits.php) 


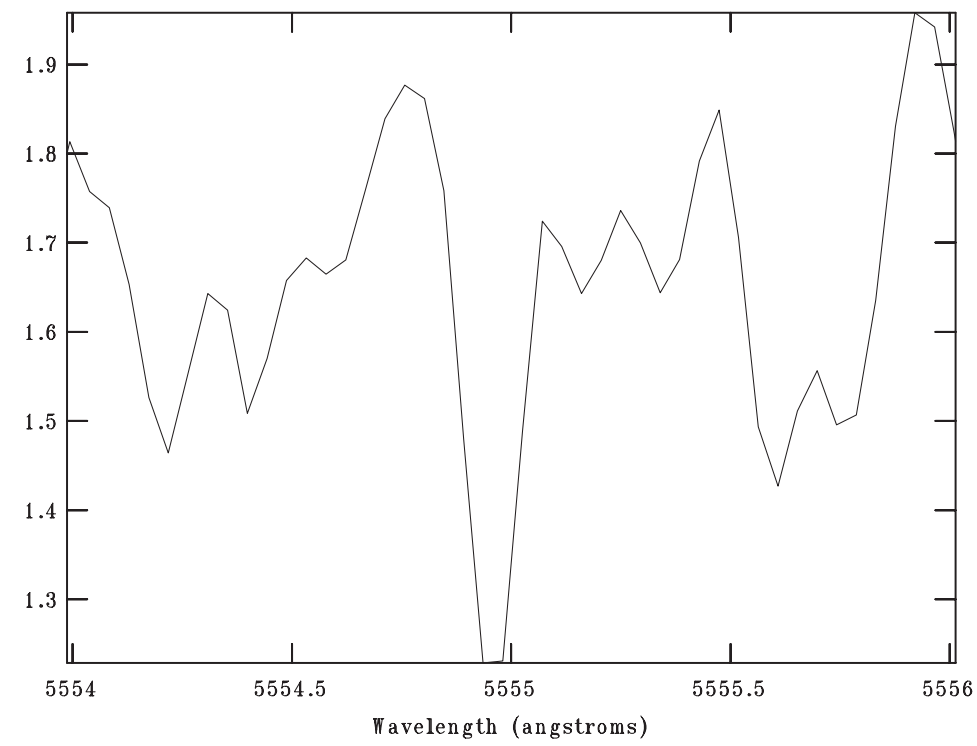

Figure 2. Part of the observed spectra of a bright star Alkaid (HIP67301), overlapped with I2 absorption features.

2008) shows that the host star has additional sodium absorptions due to the planet's atmosphere. Duplicate spectral observations on HD189733b's in and out of transit will be made to check whether small telescopes can do such challenging studies.

\section{Summary}

Transit observations of several planet host stars were made by using the 1-m reflecting and 30-cm refracting telescopes at Weihai Observatory of Shandong University, and the new high-resolution fiber-fed spectrograph (WES) has already been well installed for doing precise radial velocity measurements. Collaborations are welcome to do photometric and spectroscopic follow-up observations on exoplanet candidates.

\section{References}

Borucki, et al. 2010, Science, 327, 977

Liu, Y.-J., Sato, B., Zhao, G., \& Ando, H. 2009, Research in Astronomy and Astrophysics, 9, 1 Poddaný, S., Brát, L., \& Pejcha, O. 2010, NewA, 15, 297

Rabus, et al. 2009, A\&\&A, 508, 1011

Redfield, S., Endl, M., Cochran, W. D., \& Koesterke, L. 2008, ApJ Letter, 673, L87

Sato, B., Toyota, E., Omiya, M., et al. 2008, PASJ, 60, 1317

Wang, L., Sato, B., Zhao, G., et al. 2012, Research in Astronomy and Astrophysics, 12, 84

Zhang, et al. 2011, ChAEGA, 35, 409 